\title{
The Structure of Internal Blood Vessels in Mongolian Fetal Vertebral Bodies
}

\author{
Oyuntsetseg Demed $^{1 *}$, Yansanjav Damdinjav ${ }^{1}$, Avirmed Amgalanbaatar ${ }^{2}$, \\ Enebish Sundui ${ }^{2}$, Amgalanbaatar Dorjkhuu ${ }^{2}$ \\ ${ }^{1}$ Health Sciences University of Mongolia, Altai City, Mongolia \\ ${ }^{2}$ Health Sciences University of Mongolia, Sainshand City, Mongolia \\ Email: *oyunaa8998@yahoo.com, d.yansanjav@yahoo.com, avirmed_baba@yahoo.com, senebish@yahoo.com, \\ dorjhvvamgalanbaatar@yahoo.com
}

Received November 23, 2012; revised December 22, 2012; accepted December 30, 2012

\begin{abstract}
Purpose: Investigate specific characteristic of blood supply of fetal vertebral bodies. Material \& Methods: 16 - 30 week aged 20 fetuses (10 male, 10 female) were used for the study. Norms and requirements of Bio-Medical Ethics have not been violated in the use of human material in the study. Black-ink perfusion and cast of substance absorbing $\mathrm{X}$-ray were applied in the fetuses. Results: Three concentric zones could be distinguished in the vascular architecture of the fetal vertebral bodies. 1) The peripheral zone of perichondrial vessels; 2) The intermediate zone of radial vessels. 3. The central zone of the ossification centre vessels. We used black-ink perfusion and cast of substance absorbing X-ray were applied in the fetuses. Periosteum of fetal vertebral bodies are distributed originating from fine arteries of $70-120$ diameter and arterioles of 30 - 50 diameter which are part of arteries of network of micro blood circulation. Venules are followed along two sides of this arteriole and short precapillaries are branched out in tree-like manner from it forming capillary network surrounding arteriole and capillary plexuses. Postcapillaries branched from the network are being emerged the venules accompanied arterioles. Conclusions: Micro blood circulatory system of the vertebrae has angion structure. The peripheral zone of perichondrial vessels has combined blood supply surrounded by blindly ending capillary network drained up and down from the central zone. The central zone of vertebral body is supplied with blood by the main arteriole.
\end{abstract}

Keywords: Fetus; Vascularization; Spine; Vertebral Bodies; Intraosseous Arteries; Periosteum; X-Ray Angiography; Black-Ink Perfusion

\section{Introduction}

The vascularization of the human spine has been the subject of anatomical and micro anatomical studies for almost a century. Attention has mostly been focused on the external segmental blood supply and drainage of the vertebral bodies [1,2]. Only a few papers have described the intraosseous arteries of the vertebral bodies in detail [3-6]. Among more recent studies, a comprehensive description of both extra- and intraosseous vessels of the vertebral bodies was presented [7-11]. The latter author proposed the most convincing classification of the intraosseous arteries supplying the vertebral body, dividing them into 1) the equatorial arteries, including the nutrient arteries originating from the postcentral longitudinal anastomosis and 2 anterolateral arteries arising from the segmental artery, 2) the metaphyseal, radially disposed arteries arising from the periosteal vascular network and

${ }^{*}$ Corresponding author.
3) short, centripetal peripheral arteries originating from the same source. Almost all authors studying the vascularization of the vertebral bodies employed X-ray angiographic methods which have not permitted visualizetion of the capillary networks due to the high density of the contrast media. To investigate the situation there were used black-ink perfusion and cast of substance absorbing X-ray. The latter method offers complete visualization of the vascular system, significantly higher resolution and quasi-3-dimensional images which are especially useful in the investigation of the microvascular architecture [12,13]. It was occasionally used for investstigating the vertebral vasculature in animals including the rat and rabbit, but never in human material $[14,15]$.

In recent years the defect of spinal cord has increased in Mongolia. The purpose in our search is to decrease defect of spinal cord, the structure of the organ, to search vascularization and nerves, to define its many version. Especially Mongolian's fetus, fetus spine bone, structure 
of joint, transformation of spygmic depend on grown aged search is rare. Our search based on this direction. The search was compared Mongolian's fetus spine blood supply and other countries fetus spine blood.

\section{Material and Methods}

16 - 30 wk aged 20 fetuses (10 male, 10 female) were used for the study. Norms and requirements of BioMedical Ethics have not been violated in the use of human material in the study. Black-ink perfusion and cast of substance absorbing X-ray were applied in the fetuses.

\subsection{Black Ink Cast}

To reach the goal of defining the microcirculation, there was the black ink cast method in some hearts. But it did not reveal information about the wall structure of the heart micro blood vessels. The angiography method was used in some hearts to determine the vitelline vein such as a vertebral blood vessel and its derived branches. The first cut was performed at the level of vitelline vein. Then the cannulas were introduced into the vitelline vein. The great vessels were preventing black ink from flowing out of the vessels. Also the cannulas were put into the vitalline vein and were ligated tightly. Injection with a water suspension of black ink (1:3) was performed under mannometric control for 2 - 3 times. To improve the results, the ink was filtered three times prior to the injection. After the infusion procedure with black ink, the cannulas were removed and the vessels were ligated. After fixation in $10 \%$ formaldehyde for 21 days, dehydrated in alcohol $50^{\circ}, 60^{\circ}, 70^{\circ}, 80^{\circ}, 90^{\circ}$, for 48 hours and cleared in dimetillptalate for 72 hours the preparations were examined using a light microscope MBC-2. In this way the microcirculation of the vertebrae and intervertebral articulation were investigated.

\subsection{X-Ray Angiography Method}

The heart was isolated and immediately processed. The abdominal aorta opened, 2 - 3 glass cannulas were introduced into the lumbar arteries. The cannulas were ligated tightly in the abdominal aorta where the lumbal artery originated and connected to a plastic connector. One end of each tube was connected to a 10 - ml syringe. The vascular system was perfused with $10 \mathrm{ml}$ of $5 \%$ contrast media of (10.0 g glycerin, $\left.1.0-2.0 \mathrm{~g} \mathrm{PIO}_{2}\right)$ at $80-100$ mmHg pressure. (B. Dagdanbazar, B. Purevsuren). In some selected cases barium sulphate was used as the opaque media. Then X-ray films were taken using the URD-110 X-ray unit. The methods of opening and taking X-ray films were first described by Reiner and Schlesinger. All the procedure where performed under the control of an X-ray technician.

\section{Results}

Three concentric zones could be distinguished in the vascular architecture of the fetal vertebral bodies:

1) The peripheral zone of perichondrial vessels;

2) The intermediate zone of radial vessels;

3) The central zone of the ossification centre vessels.

The perichondrial vessels formed a relatively narrow layer from which fine arterioles were sometimes seen emerging from the inner circumference, especially from the anterolateral surface of the vertebral body, and forming a shallow, centripetally directed arcade-like array. The radial arteries originated from the segmental arteries and their branches or from the perichondrial plexus. They were regularly distributed and in the sagittal view their blindly ending branches were directed towards the vertebral end-plates. The terminal segments of these vessels showed thickened, irregular contours. Occasionally, some branches seemed to communicate with the vessels of the ossification centre. The ossification centre was mostly supplied by 2 arteries of similar size originating from the spinal branches of basivertebral artery (postcentral anastomosis), entering the vertebral body from the posterior aspect and extensively branching into a lenticular network with a fan-shaped, centrifugal arrangement of the vessels. The intervertebral spaces and end-plate regions were completely devoid of blood vessels, terminated at the bone/cartilage interface, forming blind buds or, occasionally, irregular loops. Periosteum of fetal vertebral bodies are distributed originating from fine arteries of 70 - 120 diameter and arterioles of 30 - 50 diameter which are part of arteries of network of micro blood circulation. Venules are followed along two sides of this arteriole and short precapillaries are branched out in tree-like manner from it forming capillary network surrounding arteriole and capillary plexuses. Postcapillaries branched from the network are being emerged the venules accompanied arterioles. However, in this micro blood circulation of vertebral body split film and ring shaped arrangement in the center of which capillary network should be localized, has not been seen clearly. It is observed that in few cases half of the module has been seen (Figure 1).

\section{Discussion}

The general architecture of the blood vessels present in the vertebral bodies of fetuses from the 2nd trimester shows the pattern observed by other authors. Although some observations of the fetal vertebrae were mentioned in works $[2,4,6]$. However, they investigated fetuses from the 3rd trimester and at term. It seems therefore that differences observed in the present study stem from the earlier stage of fetal development that was studied. In dye-injected specimens, the localisation and the mode of branching of the radial arteries permitted their identifica- 


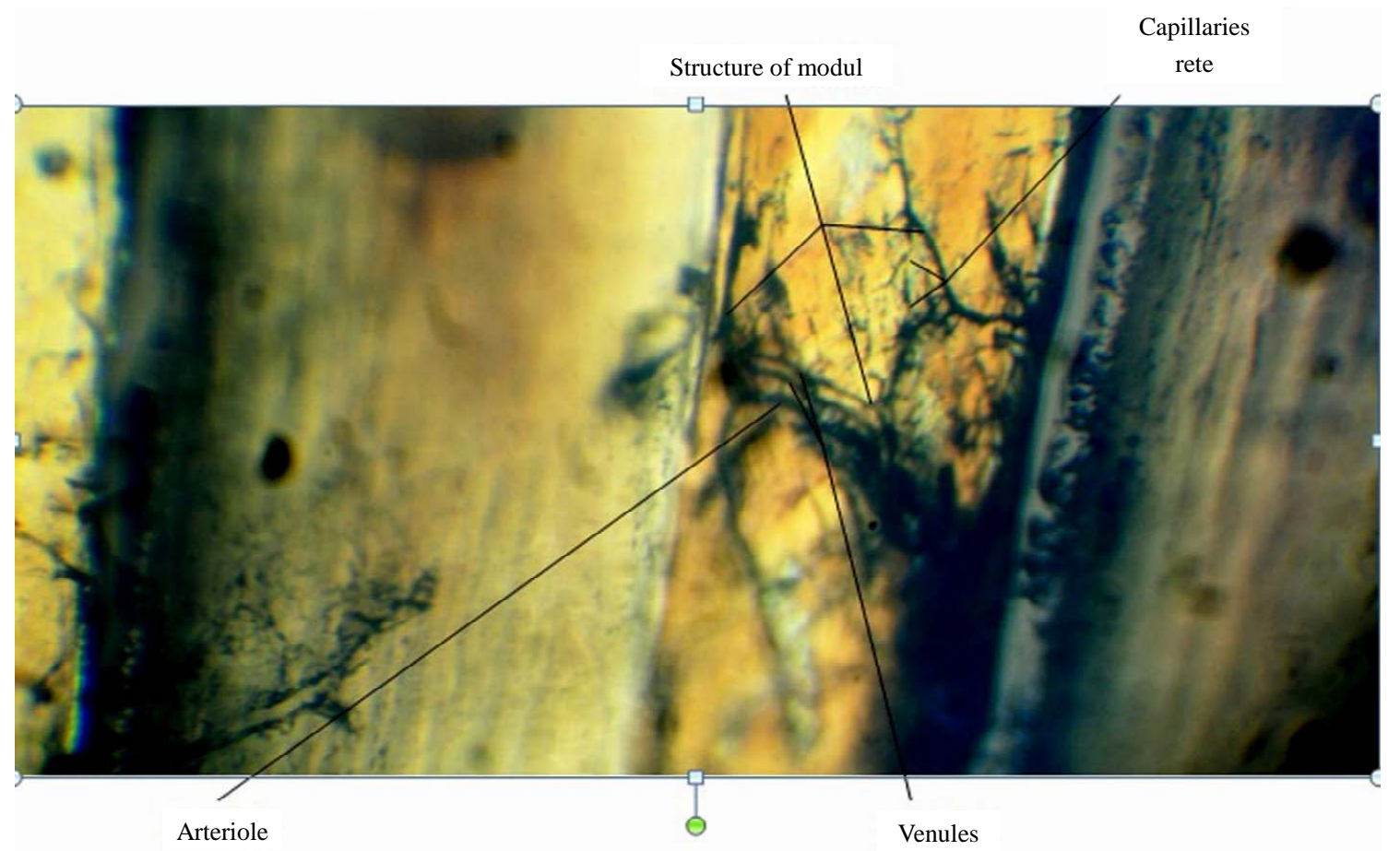

Figure 1. Photomicrograph of artery network in fetal vertebral body.

tion as the metaphyseal arteries, according to the classification of Ratcliffe, while the occasional shallow arcades protruding from the perichondrial network correspond as far as their size and arrangement is concerned to the peripheral vessels. The equatorial vessels entering the vertebral body from an anterolateral direction and communicating with the vasculature of the ossification centre were found only rarely [16].

Since the interconnections between the metaphyseal arteries reported by Ratcliffe could not be seen in our material, it seems that in the 2nd trimester these arteries are at the stage of ingrowth into the vertebral body cartilage. They become fully incorporated into the ossification centre about the 36th wk of fetal life [10]. Occasional arterioles entering the ossification centre from the periphery probably represent the antero-lateral equatorial vessels, sometimes seen also in dye-injected specimens. However, they did not occur consistently at the developmental stage investigated in this study, although they had been described in 7 - 10 mo old fetuses $[4,6,10]$. The fold-like capillary plexuses with their regular distribution around the inner perimeter of the vertebral body may be precursors of the later peripheral vessel system. Ratcliffe did not find peripheral arteries in his material but it seems that the development of peripheral vessels may just begin in the 2nd trimester, albeit only in some local sectors. The vasculature of the ossification centre clearly shows that the posterior vessels are mainly, if not exclusively, responsible for the supply and drainage of the centre in the 2nd trimester.

In our material, most of the vertebral bodies had 2 nutrient arteries, while the others were supplied by just 1 . These results are in agreement with the observations of Ratcliffe.

The presence of arterial sphincters has not yet been reported in the intraosseous vasculature of human vertebral bodies, but such sphincters, both arterial and venous, were described in the rat [14]. The appearance of the capillary vessels at the advancing border of the ossification centre is similar to that of the "vascular besom" described by us in corrosion casts of the femoral vessels directed towards the metaphyseal plate and it seems to be a typical arrangement of capillaries at the bone/cartilage interface in the ossification areas [16]. The internal venous sys- tem of the fetal vertebral bodies generally followed the course of the arteries, as also seen in adults, although some specific venous arrangements such as the subchondral network and the horizontal collecting vein of the endplate region described by Crock et al. have not yet been developed $[7,8]$.

Only Ferguson mentioned the presence of blood vessels in the region of fetal end-plates and even of the annulus fibrosus. Our observations, however, agree with those of Guida et al. and Ratcliffe who also found the end-plate area avascular. A striking observation was the axial avascular area in the centre of the vertebral bodies of the youngest fetus examined, corresponding to the location of the notochord and making the vasculature of 
the ossification centre ring-shaped. According to our knowledge, this is the first description of such vascular array in the fetal vertebral body. The notochord disappears completely on the turn of the 1st trimester, while the earliest ossification centres in the vertebral bodies have been observed as early as in the 8-wk fetuses [17]. It seems therefore that the ossification centre is first formed around the remnants of the notochord and later at the beginning of the 2nd trimester the vessels advance in both directions: centrifugally, increasing the size of the centre, and centripetally, invading the formerly avascular central region. The observed arterial arcades located medially to the advancing border of the ossification centre might be the manifestation of the initial ring-shaped arrangement of the centre. It can be speculated that the notochord cells may contain some angiogenesis-inhibiting factors acting locally on the developing vessels of the ossification centre. Delayed vascular penetration and, subsequently, ossification of that region can probably lead to such rarely occurring congenital malformations of the vertebral bodies as the persistent notochord canal or even cleft vertebral body [18]. Taken together, the observations on 2nd trimester fetuses (this study), 3rd trimester fetuses and infants (Ratcliffe) as well as adults (Crock, Ratcliffe,) suggest a biphasic development of the internal (intrachondral and intraosseous) vascular architecture of the human vertebral body. In the fetal and early infantile period, the vessels grow centripetally from the periphery and centrifugally from the ossification centre, until they meet and form an integrated, extensively anastomosing system. Afterwards, during late childhood and adolescence, the radial vessels are gradually withdrawn and the anastomoses disappear, dividing the vertebral body into separate compartments supplied and drained by individual arterio-venous pairs.

\section{Conclusions}

Three concentric zones could be distinguished in the vascular architecture of the fetal vertebral bodies:

- The peripheral zone of perichondrial vessels;

- The intermediate zone of radial vessels;

- The central zone of the ossification centre vessels.

Micro blood circulatory system of the vertebrae has angion structure. The peripheral zone of perichondrial vessels has combined blood supply surrounded by blindly ending capillary network drained up and down from the central zone. The central zone of vertebral body is supplied with blood by the main arteriole.

\section{Acknowledgements}

I strongly appreciate to everyone that helped my research work. Special thanks to Sc.D and Prof., academician Amgalanbaatar D., Sc.D and Prof. in Biology, Enebish S.,
MD. Avirmed A., MScD Dagdanbazar B. of Anatomy and Morphology Department, Bio-Medical School, Health Science University of Mongolia.

Also let me express my gratitude to all the colleagues, Department lab of Microbiology and Immunology, Traditional Medicine School, Foreign Language Department instructor, Aldarmaa T. of HSUM and Medical College of Gobi-Altai province.

Allow me to wish all above mentioned associates the best in their further researches and long lasting happy life.

\section{REFERENCES}

[1] R. S. Harris and D. M. Jones, "The Arterial Supply of the Adult Cervical Vertebral Bodies,” Journal of Bone and Joint Surgery, Vol. 38B, No. 4, 1956, pp. 922-929.

[2] T. A. Willis, "Nutrient Arteries of the Vertebral Bodies," Journal of Bone and Joint Surgery, Vol. 31A, No. 3, 1949, pp. 538-540.

[3] G. Wagoner and E. P. Pendergrass, "Intrinsic Circulation of the Vertebral Body. With Roentgenologic Considerations,” American Journal of Roentgenology, Vol. 27, 1932, pp. 818-826.

[4] W. R. Ferguson, "Some Observations on the Circulation in Fetal and Infant Spines," Journal of Bone and Joint Surgery, Vol. 32A, No. 3, 1950, pp. 640-648.

[5] A. M. Wiley and J. Trueta, "The Vascular Anatomy of the Spine and Its Relationship to Pyogenic Vertebral Osteomyelitis," Journal of Bone and Joint Surgery, Vol. 41B, No. 4, 1959, p. 796-809.

[6] G. Guida, F. Cigala and V. Riccio, "The Vascularization of the Vertebral Body in the Human Fetus at Term," Clinical Orthopaedics, Vol. 65, pp. 229-234.

[7] H. V. Crock, H. Yoshizawa and S. K. Kame, “Observations on the Venous Drainage of the Human Vertebral Body,” Journal of Bone and Joint Surgery, Vol. 55B, No. 3, 1973, pp. 528-533.

[8] H. V. Crock and H. Yoshizawa, "The Blood Supply of the Lumbar Vertebral Column,” Clinical Orthopaedics, Vol. 115, No. 1, 1976, pp. 6-21.

[9] J. F. Ratcliffe, "The Arterial Anatomy of the Adult Human Lumbar Vertebral Body: A Microarteriographic Study," Journal of Anatomy, Vol. 131, No. 1, 1980, pp. 57-79.

[10] J. F. Ratcliffe, “The Arterial Anatomy of the Developing Human Dorsal and Lumbar Vertebral Body: A Microarteriographic Study,” Journal of Anatomy, Vol. 133, No. 4, 1981, pp. 625-638.

[11] J. F. Ratcliffe, “An Evaluation of the Intra-Osseous Arterial Anastomoses in the Human Vertebral Body at Different Ages: A Microarteriographic Study,” Journal of Anatomy, Vol. 134, No. 2, 1982, pp. 373-382.

[12] A. Lametschwandtner, A. Miodonski and P. Simonsberger, "On the Prevention of Specimen Charging in Scanning Electron Microscopy of Vascular Corrosion Casts by Attaching Conductive Bridges,” Mikroskopie, Vol. 36, No. 9-10, 1980, pp. 270-273. 
[13] A. Lametschwandtner, U. Lametschwandtner and T. Weiger, "Scanning Electron Microscopy of Vascular Corrosion Casts-Technique and Application: Updated Review," Scanning Microscopy, Vol. 4, No. 4, 1990, pp. 889-941.

[14] M. A. Konerding and M. Blank, "The Vascularization of the Vertebral Column of Rats," Scanning Microscopy, Vol. 1, No. 4, 1987, pp. 1727-1732.

[15] S. Oki, Y. Matsuda, T. Itoh, T. Shibata, H. Okumura and J. Desaki, "Scanning Electron Microscopic Observations of The Vascular Structure of Vertebral End-Plates in Rabbits,” Journal of Orthopaedic Research, Vol. 12, No. 3, 1994, pp. 447-449. doi:10.1002/jor.1100120318

[16] A. Skawina, J. A. Litwin, J. Gorczyca and A. J. Miodon- ski, "Blood Vessels in Epiphyseal Cartilage of Human Fetal Femoral Bone: A Scanning Electron Microscopic Study of Corrosion Casts," Anatomy and Embryology, Vol. 189, No. 5, 1994, pp. 457-462. doi:10.1007/BF00185441

[17] R. Bareggi, V. Grill, M. Zweyer, P. Narducci and A. Forabosco, "A Quantitative Study on the Spatial and Temporal Ossification Patterns of Vertebral Centra and Neural Arches and Their Relationship to the Fetal Age,” Annals of Anatomy, Vol. 176, No. 4, 1994, pp. 311-317. doi:10.1016/S0940-9602(11)80502-9

[18] R. N. Hensinger and C. MacEwan, "Congenital Anomalies of the Spine,” The Spine, Vol. 1, 1975, pp. 157-229. 